\title{
Aspectos paleográficos de um processo crime de roubo e estupro do início do século XX: análise das abreviaturas
}

\author{
Palaeographic study of a crime of robbery \\ and rape of the early 20th century: an analysis of abbreviations
}

\author{
Damares Oliveira de Souza* \\ Universidade Estadual de Feira de Santana, Feira de Santana, BA, Brasil \\ Rita de Cássia Ribeiro de Queiroz ${ }^{* *}$ \\ Universidade Estadual de Feira de Santana, Feira de Santana, BA, Brasil
}

\begin{abstract}
Resumo: Os processos crimes são documentos jurídicos que possuem vastos e ricos mananciais de informações, e constituem fonte de conhecimento e objeto de estudo para pesquisadores de áreas diversas. Incursionar pelouniverso filológicopaleográfico é uma atividade que, além da preservação do suporte, possibilita a leitura e interpretação dos documentos, bem como de suas formas gráficas. Sendo assim, neste artigo, objetivamos apresentar o estudo de alguns aspectos paleográficos de um processo crime de roubo e estupro, do início do século XX, arquivado no Centro de Documentação e Pesquisa (CEDOC), localizado na Universidade Estadual de Feira de Santana (UEFS), no Estado da Bahia, assim descrito: série: processo crime, sessão: judiciária, sob a localização: estante 03 , Caixa 65, Documento 1205; tendo como suportes as suas edições fac-similar e semidiplomática. Utilizamos como aportes teóricos Spina (1977), Acioli (2003), Spaggiari e Perugi (2004), Cambraia (2005) e Queiroz (2007), dentre outros.
\end{abstract}

Palavras-chave: Processo crime. Paleografia. Edição fac-similar. Edição semidiplomática.

\begin{abstract}
Criminal processes are legal documents that preserve vast and rich sources of information, constitute sources of knowledge and objects of study by researchers from diverse areas. Incursing through the philological-palaeographic universe is an activity that, besides preservation, makes possible the reading and interpretation of the documents, as well as their graphic forms. Thus, in this article, we present the study of some palaeographic aspects of a crime process of robbery and rape, from the beginning of the $20^{\text {th }}$ century, that is archived at the Documentation and Research Center (CEDOC), at the State University of Feira de Santana (UEFS), in the State of Bahia, as follows: series: crime process, session: judicial, under the location: shelf 03, Box 65, Document 1205; the study is based on its facsimile and semidiplomatic editions. We consulted as theoretical bases: Spina (1977), Acioli (2003), Spaggiari and Perugi (2004), Cambraia (2005) and Queiroz (2007).
\end{abstract}

Keywords: Criminal process. Palaeography. Fac-similar edition. Semidiplomatic edition.

\footnotetext{
* Mestre em Estudos Linguísticos pela Universidade Estadual de Feira de Santanta - UEFS, Feira de Santana, BA, Brasil; da_mares.oliveira@hotmail.com

** Professora Titular Pleno, Departamento de Letras e Artes, Universidade Estadual de Feira de Santanta - UEFS, Feira de Santana, BA, Brasil; rcrqueiroz@uol.com.br
} 


\section{CONSIDERAÇÕES INICIAIS}

Levando em consideração o valor dos documentos escritos, tivemos como intuito desenvolver um estudo de cunho filológico e paleográfico do processo crime de roubo e estupro de Maria Francisca e Maria dos Santos, ocorrido na cidade de Feira de Santana, Bahia, no início do século XX.

Assim sendo, objetivamos apresentar, neste artigo, o estudo de alguns aspectos paleográficos do referido processo crime, a partir de teóricos como Spina (1977), Acioli (2003), Cambraia (2005), dentre outros, tendo por suporte o exercício da Crítica Textual, especificamente, na realização das edições fac-similar e semidiplomática. Neste segmento, destacamos que este trabalho está vinculado à dissertação de mestrado Edição filológica e estudo lexical de um processo crime de roubo e estupro do inicio do século XX, defendida em março de 2018, junto ao Programa de Pós-Graduação em Estudos Linguísticos, da Universidade Estadual de Feira de Santana - BA.

\section{EDIÇÃO DO PROCESSO CRIME DE ROUBO E ESTUPRO}

Trata-se de um documento jurídico, lavrado em 1914, o qual encontra-se arquivado no Centro de Documentação e Pesquisa (CEDOC), localizado na Universidade Estadual de Feira de Santana - BA, sob a cota: Estante 03, Caixa 65, Documento 1205.

As vítimas são Maria Francisca de Jesus e Maria dos Santos. Segundo consta nos autos, os crimes foram cometidos enquanto Maria Francisca de Jesus dirigia-se para a igreja, na madrugada de 24 para 25 de dezembro do ano de 1913, objetivando assistir à missa do "Natal", e deixou dormindo em sua casa a filha de nove anos de idade, Maria dos Santos. Ao voltar, Maria Francisca deparou-se com o arrombamento de portas e janelas da frente de sua casa, roubo de muitos objetos e, finalmente, o estupro de sua filha Maria dos Santos, que foi acordada pelo ladrão para aquele fim, o qual chegou ameaçando-a de morte. $\mathrm{O}$ acusado de ter cometido os crimes de roubo e estupro é identificado como Possidonio Gasparino de Araújo, pessoa conhecida da vítima, pois a mesma declarou tê-lo reconhecido pela voz, informando, ainda, que este já vinha rondando a frente de sua casa.

\subsection{Edições fac-similar e semidiplomática}

A edição de texto pode ser considerada como uma das formas mais importantes para a salvaguarda e/ou preservação de documentos, além de torná-los mais acessíveis ao público. Neste sentido, existem diversos tipos de edição, cada uma com características próprias e definidas, das quais a escolha do tipo a ser realizada depende do próprio documento e do público destinado.

Sendo assim, em virtude de o corpus deste trabalho constituir-se de um documento jurídico, em um único registro, optamos pela realização das edições facsimilar, isto é, pela reprodução fotográfica do texto, onde não se permite a intervenção do crítico textual - o que contribui para a não-deterioração do documento, evitando, assim, o seu manuseio - e a edição semidiplomática, por esta possuir um grau mínimo 
de intervenção (Cambraia, 2005).

Destarte, para a realização das edições fac-similada e semidiplomática, fizemos fotografias digitais de todo o processo crime e adotamos alguns critérios de transcrição, os quais apresentamos a seguir.

\subsubsection{Critérios adotados na descrição e transcrição semidiplomática}

Para a edição semidiplomática, foram seguidos alguns critérios desenvolvidos e utilizados pelos estudiosos do $\mathrm{NEMa}^{1}$ e pesquisadores do $\mathrm{GET}^{2}$, correlacionados com os de Queiroz (2007, p. 34), a saber:

- Para a descrição, observamos:

1) Número de colunas;

2) Número de linhas da mancha escrita;

3) Existência de ornamentos;

4) Maiúsculas mais interessantes;

5) Existência de sinais especiais;

6) Número de abreviaturas;

7) Tipo de escrita;

8) Tipo de papel;

9) Data do manuscrito.

- Para a transcrição, optamos por:

a) Respeitar fielmente o texto: grafia (letras e algarismos), linha, fólio etc.;

b) Indicar o número do fólio à margem direita;

c) Numerar o texto linha por linha, indicando a numeração de cinco em cinco, desde a primeira linha do papel almaço. A numeração é não corrida, feita fólio a fólio.

d) Separar as palavras unidas e unir as separadas;

e) Desdobrar as abreviaturas, apresentando-as em itálico e negrito;

f) Utilizar colchetes para as interpolações: [];

g) Indicar as rasuras, acréscimos e supressões através dos seguintes operadores: (†) ) rasura ilegível; [†] escrito não identificado; // leitura conjecturada; $<>$ supressão; () rasura ou mancha; [] acréscimo; ** interferências de terceiros.

h) Para a transcrição utilizamos a fonte Times New Roman, tamanho 11.

\footnotetext{
${ }^{1}$ Núcleo de Estudos do Manuscrito - Universidade Estadual de Feira de Santana.

2 Grupo de Edição de Textos - composto por pesquisadores da área de Filologia, Linguística, Antropologia, Desenho e outras; coordenado pela professora Doutora Rita de Cássia Ribeiro de Queiroz.
} 


\subsection{2 À guisa de ilustração: edição de um fólio do processo}

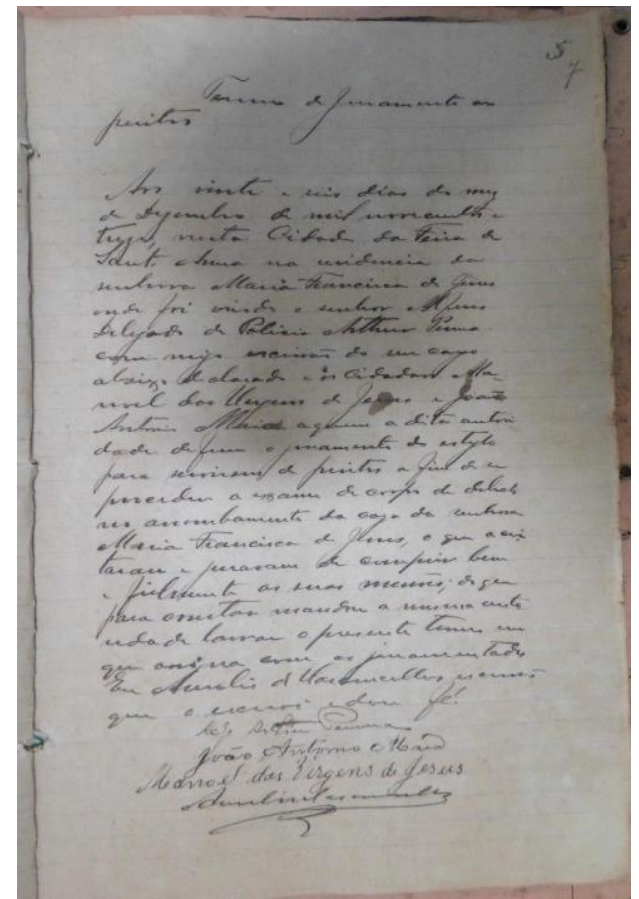

Figura 1 - Fól. 8r
Termo de juramento aos

Peritos

Aos vinte e seis dias do mez de Dezembro mil novecentos e treze, nesta cidade da Feira de Sant'Anna na residencia da senhora Maria Francisca de Jesus onde foi vindo o senhor Alferes Delegado de Policia Arthur Penna com migo escrivão do seu cargo abaixo declarado e os cidadaos Manoel das Virgens de Jesus e João Antonio Maia a quem a dita autori dade deferiu o juramento do estylo para servirem de peritos a fim de se proceder a exame de corpo de delicto no arrombamento da caza da senhora

20 Maria Francisca de Jesus, o que aceitaram e juraram de cumprir bem e fielmente as suas missões; do que para constar mandou a mesma autoridade lavrar o presente termo que assigna com os juramentados Eu Aurelio d[e] Vasconcellos escrivão que o escrevi e dou fé.

Alferes Arthur Penna João Antonio Maia
Manoel das Virgens de Jesus AurelioVasconcelos

\subsection{Paleografia: Descrição dos aspectos extrínsecos e intrínsecos do documento}

Entendemos que a edição crítica é uma operação extremamente necessária à completa interpretação filológica e, para que o exercício da Crítica Textual possa efetivar-se por completo, é indispensável o reconhecimento do seu caráter transdisciplinar, uma vez que, para fixar textos, isto é, estabelecer, preparar e editar os mesmos, é necessário o auxílio de outras disciplinas de outras áreas do conhecimento.

Considerando a sua grande importância sobre o labor do crítico textual, destacamos a Paleografia como disciplina essencial no estudo de documentos, pois, em sentido lato, proporciona um amplo conhecimento sobre os manuscritos e mais segurança quanto a sua autenticidade, uma vez que, de acordo com a etimologia grega da palavra: paleos (antiga) + graphein (escrita) (Berwanger, Leal, 2008, p. 16), a Paleografia é compreendida como o estudo de escritas antigas.

De acordo com Spaggiari e Perugi (2004), a Paleografia tem como propósito o estudo dos caracteres gráficos, examinando e catalogando as formas de escrita. 
Todavia, "[...] não cabe ao paleógrafo somente ler textos; a ele compete igualmente datá-los, estabelecer sua origem e procedência e criticá-los quanto à sua autenticidade, levando em consideração o aspecto gráfico dos mesmos" (Acioli, 2003, p. 5).

Dessa maneira, a Paleografia apresenta finalidade tanto teórica quanto pragmática, de modo que a primeira tem como objetivo a preocupação em entender como se construíram sócio-historicamente os processos de escrita, enquanto a segunda busca a habilitação de leitores modernos para avaliarem a autenticidade de um documento e interpretarem corretamente as escritas do passado (Cambraia, 2005). Portanto, "[...] a Paleografia abrange a história da escrita, a evolução das letras, bem como os instrumentos para escrever. Pode ser considerada arte ou ciência. É ciência na parte teórica. E arte na aplicação prática. Porém, acima de tudo, é uma técnica". (Berwanger, Leal, 2008, p. 16).

\subsubsection{Aspectos paleográficos}

Os aspectos paleográficos proporcionam informações valiosas para a realização de diversos estudos, como, por exemplo, o estudo dos caracteres gráficos que favorece o processo de edição e análise do texto. Com tal intento, atemo-nos aos aspectos materiais, tais como o suporte da escrita, dimensão do documento, estado de conservação, tinta, datação, quantidade de páginas, entre outras características, dado que "a Paleografia tem por objeto o estudo das características extrínsecas dos documentos, admitindo a sua leitura, transcrição e determinação de sua data e origem". (Berwanger, Leal, 2008, p. 16).

O processo crime estudado, lavrado em 1914, com 56 fólios, está escrito em papel almaço pautado, com a seguinte dimensão: $225 \mathrm{~mm}$ x $331 \mathrm{~mm}$; encontra-se em bom estado de conservação. Ademais, os fólios, em sua maioria numerados, estão amarrados por um barbante que os costura. Vejamos a seguir outras particularidades do documento:

Quadro 1 - Principais características extrínsecas do suporte

\begin{tabular}{|c|c|c|}
\hline $\begin{array}{l}\text { CARACTERÍSTICAS } \\
\text { EXTRÍNSECAS }\end{array}$ & FÓLIOS & EXEMPLOS \\
\hline Carimbo & $1 \mathrm{r}$ & Figuras 2 e 3 \\
\hline Ficha catalográfica & $\begin{array}{c}1 \mathrm{r} \\
\text { (Sobre a capa do } \\
\text { processo) }\end{array}$ & Figura 4 \\
\hline Marca d'água "Almasso" & $\begin{array}{l}\text { f.3, f.10, f. } 24, \text { f. } 27, \\
\text { f. } 29 \text {, f. } 31, \text { f.39, f. } 40, \\
\text { f. } 42, \text { f. } 46, \text { f. } 48, \text { f. } 50\end{array}$ & Figura 5 \\
\hline Marca d'água "Superiore" & f.41, f. 43, f.47, f.51 & Figura 6 \\
\hline Marca d'água "Fiume" & $\begin{array}{l}\text { f. } 2 \text {, f. } 9, \text { f. } 23, \text { f. } 25, \\
\text { f. } 26, \text { f. } 28, \text { f. } 30, \text { f. } 32, \\
\text { f. } 37\end{array}$ & Figura 7 \\
\hline Marca d'água “A. Binda \& C & f.1, f.52 e f.53. & Figura 8 \\
\hline $\begin{array}{c}\text { Marca d'água "Almasso Extra } \\
\text { Lambrensis" }\end{array}$ & $\begin{array}{l}\text { f. } 54, \text { f. } 55, \text { f.56 e a } \\
\text { contracapa. }\end{array}$ & Figura 9 \\
\hline Rasgos & $\begin{array}{c}12 \mathrm{v}, 13 \mathrm{r}, 13 \mathrm{v}, 14 \mathrm{v} \mathrm{e} \\
15 \mathrm{v} .\end{array}$ & Figuras 10 e 11 \\
\hline
\end{tabular}




\begin{tabular}{|c|c|c|}
\hline Manchas de tinta & $\begin{array}{c}\text { 8r, 19v, 32r, 40r, } \\
40 \mathrm{v}, 41 \mathrm{r}, 43 \mathrm{v}\end{array}$ & Figuras 12 e 13 \\
\hline Marcações de lápis de cera de cor azul & $15 \mathrm{r}, 16 \mathrm{r}, 17 \mathrm{r}, 18 \mathrm{v}$, & Figuras 14 \\
& $19 \mathrm{r}, 28 \mathrm{v}$ & \\
\hline Marcações de lápis de cera de cor & $1 \mathrm{r}, 12 \mathrm{r}, 13 \mathrm{v}, 16 \mathrm{v}$, & Figuras 15 e 16 \\
vermelha & $\begin{array}{c}17 \mathrm{r}, 17 \mathrm{v}, 18 \mathrm{r}, 19 \mathrm{v}, \\
\end{array}$ & \\
\hline Sem numeração & $30 \mathrm{r}, 28 \mathrm{r}, 29 \mathrm{r}, 30 \mathrm{r}$ & \\
\hline & $51 \mathrm{r}, 47 \mathrm{r}, 48 \mathrm{r}, 49 \mathrm{r}, 50 \mathrm{r}$, & Figuras 17 \\
& $54 \mathrm{r}, 55 \mathrm{r}$, & \\
\hline
\end{tabular}

Fonte: Processo crime de roubo e estupro de Maria Francisca de Jesus e Maria dos Santos, 1914. Elaboração: Damares Oliveira de Souza.
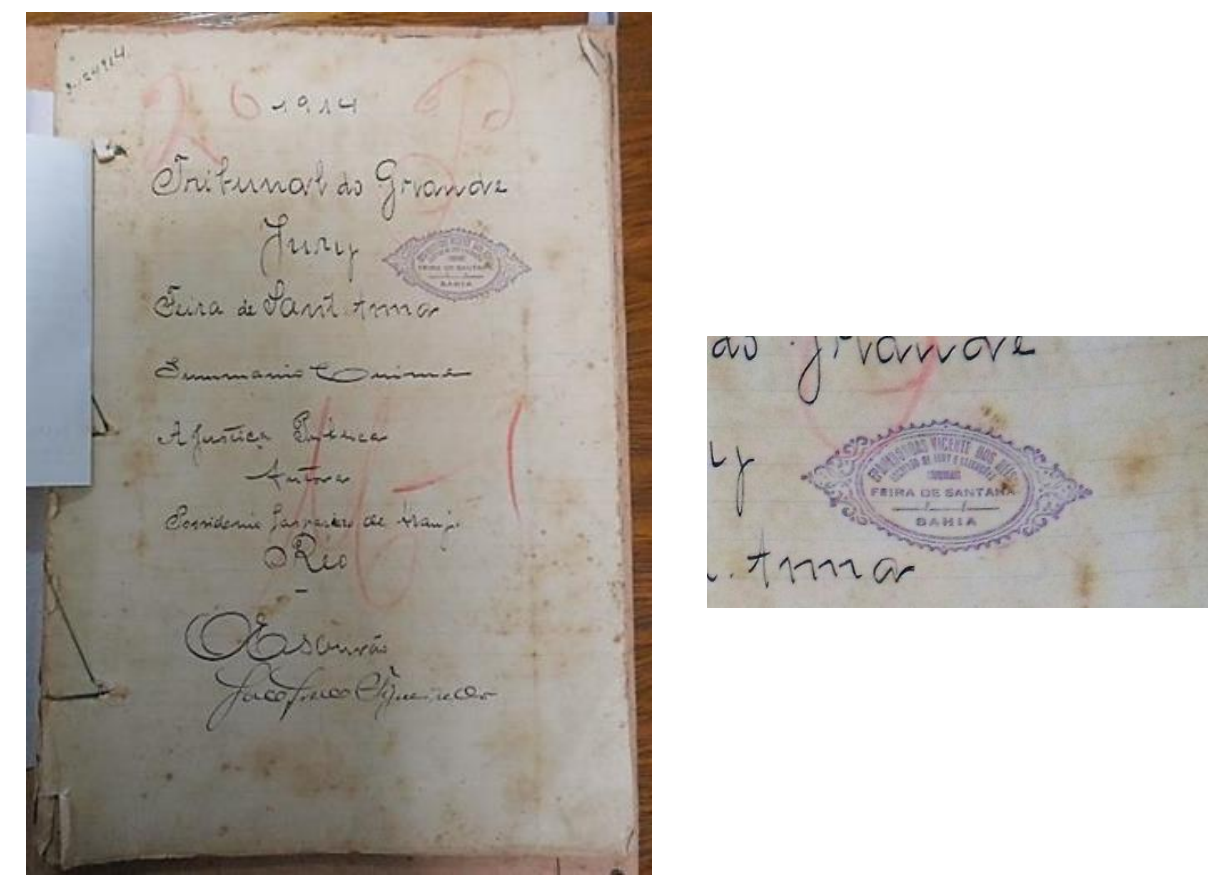

Fonte: Processo crime de roubo e estupro de Maria Francisca de Jesus e Maria dos Santos, 1914/ CEDOC / UEFS. Fotografia: Damares Oliveira de Souza.

Figuras 2 e 3 - Fól. 1r: Destaque para o carimbo ${ }^{3}$ 


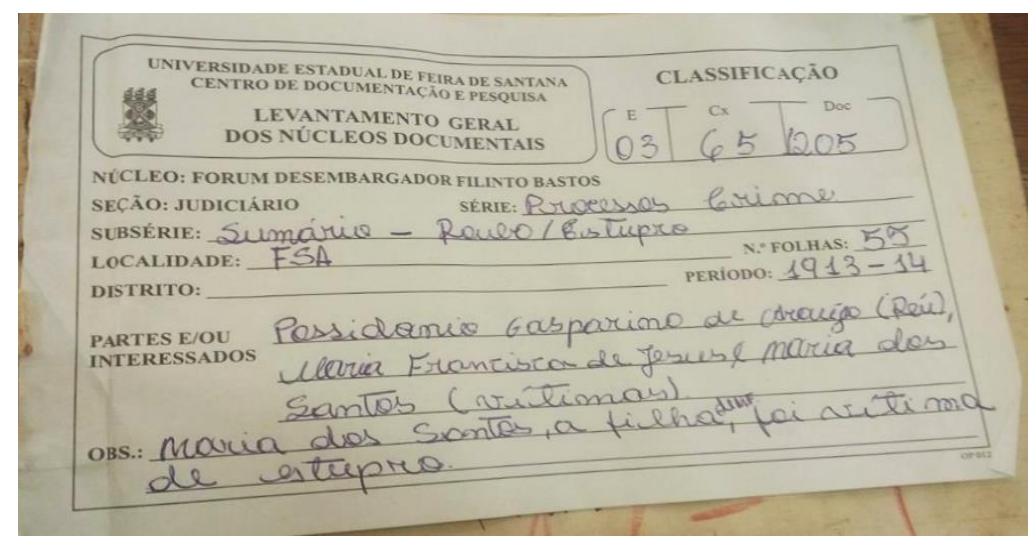

Fonte: Processo crime de roubo e estupro de Maria Francisca de Jesus e Maria dos Santos, 1914/ CEDOC / UEFS.

Fotografia: Damares Oliveira de Souza.

Figura 4 - Fól. 1r: Capa do processo

$\mathrm{Na}$ ficha catalográfica constam as seguintes informações: o nome do Fórum Desembargador Filinto Bastos; o Centro de Documentação e Pesquisa, responsável pela catalogação e guarda dos documentos; a localização do documento ( $\mathrm{E}$ = estante, $\mathrm{Cx}=$ caixa e Doc = documento); a seção: judiciária; a série: Processos crime; a subsérie: Sumário - Roubo / Estupro; a localidade: Feira de Santana; o número de folhas: 55 (exceto a capa); o período: 1913 - 1914; as partes interessadas: Possidonio Gasparino de Araújo, o réu do processo, Maria Francisca de Jesus e Maria dos Santos, as partes autoras; e as observações: Maria dos Santos, a filha (de Maria Francisca), foi vítima de estupro.

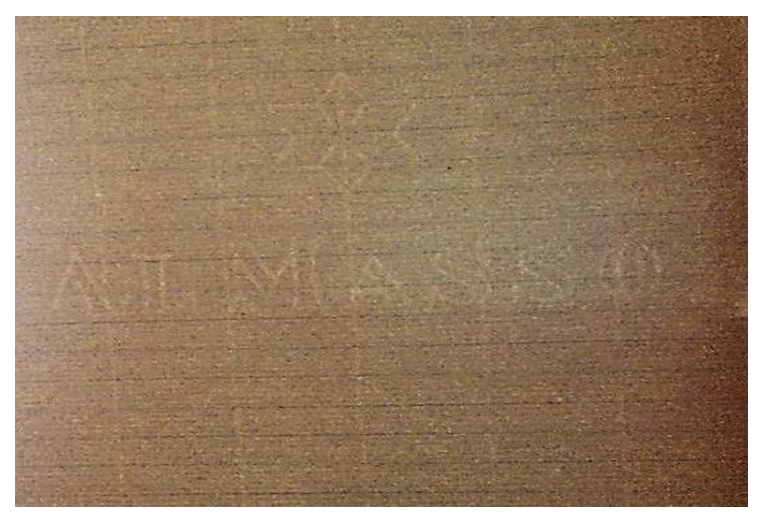

Fonte: Processo crime de roubo e estupro de Maria Francisca de Jesus e Maria dos Santos, 1914/ CEDOC / UEFS.

Fotografia: Damares Oliveira de Souza.

Figura 5 - Fól. 50: Marca d’água "ALMASSO” 

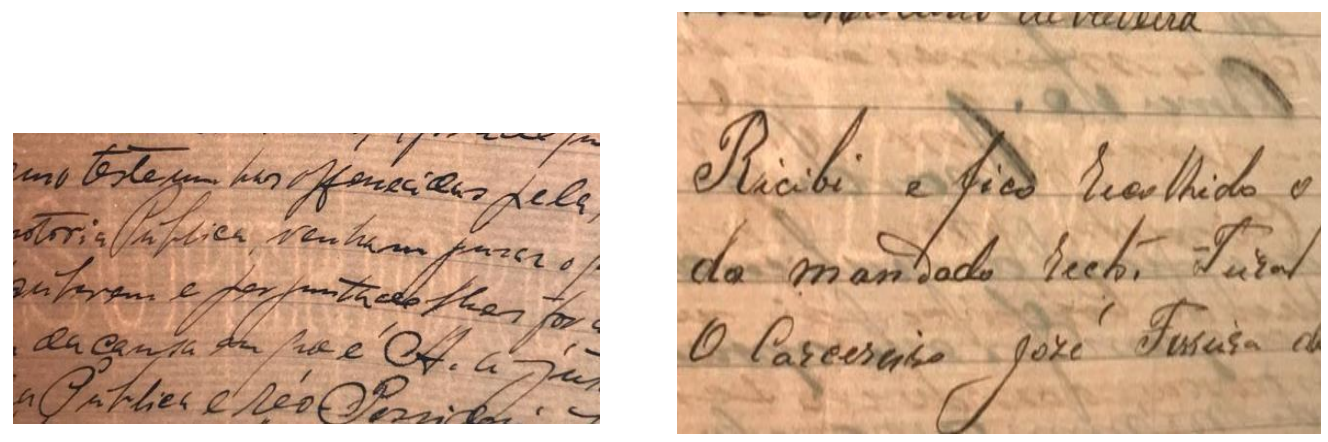

Fonte: Processo crime de roubo e estupro de Maria Francisca de Jesus e Maria dos Santos, 1914/ CEDOC / UEFS. Fotografia: Damares Oliveira de Souza.

Figura 6 - Fól. 51: Marca d'água "SUPERIORE"

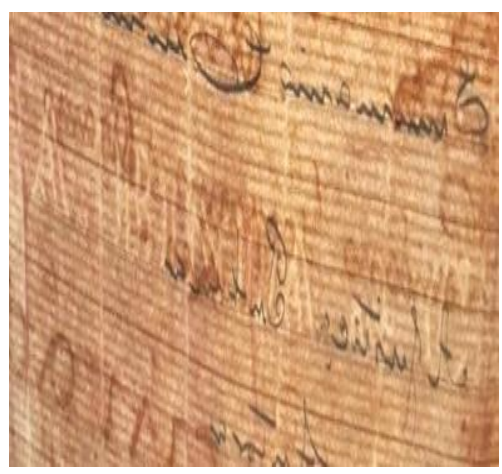

Figura 7 - Fól. 23: Marca d'água "FIUME"

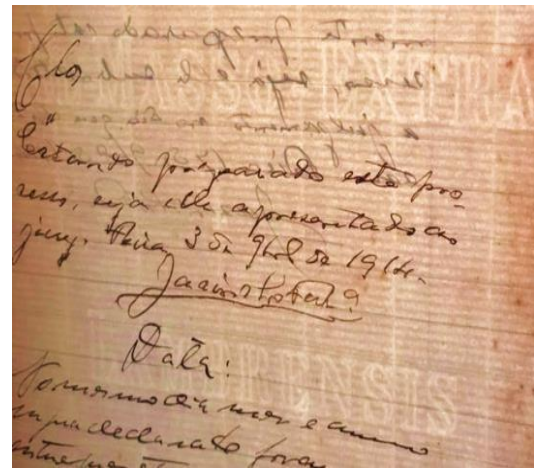

Fonte: Processo crime de roubo e estupro de Maria Francisca de Jesus e Maria dos Santos, 1914/ CEDOC / UEFS.

Fotografia: Damares Oliveira de Souza.

Figura 8 - Fól. 1: Marca d'água "A. Binda \& C"

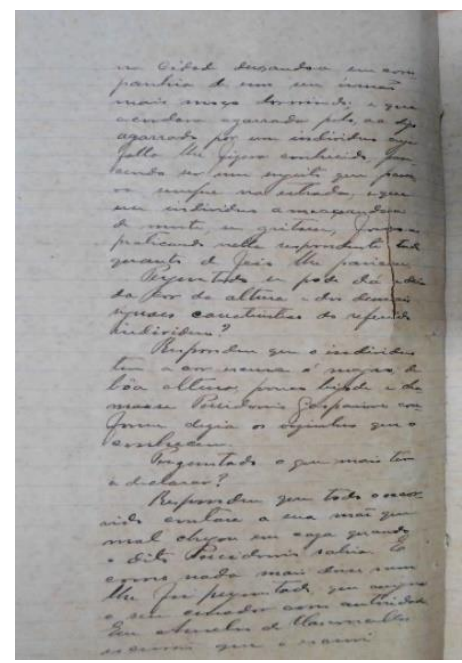

Figura 9- Fól. 56: Marca d'água "Almasso Extra Lambriensis"

Fonte: Processo crime de roubo e estupro de Maria Francisca de Jesus e Maria dos Santos, 1914 / CEDOC / UEFS.

Fotografia: Damares Oliveira de Souza.

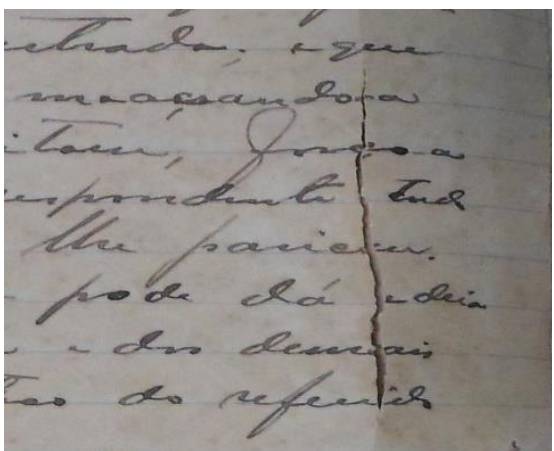

Figuras 10 e 11 - Fól. 12v: Destaque para o rasgo 

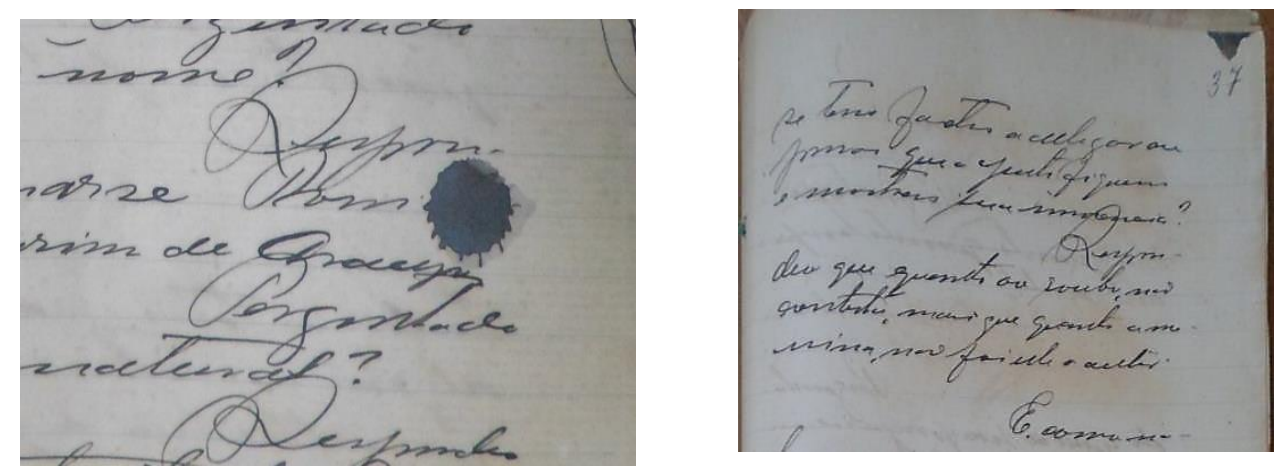

Fonte: Processo crime de roubo e estupro de Maria Francisca de Jesus e Maria dos Santos, 1913 / CEDOC / UEFS. Fotografia: Damares Oliveira de Souza.

Figuras 12 e 13 - Fól. 40r e f. 41r: Destaque para as manchas de tinta

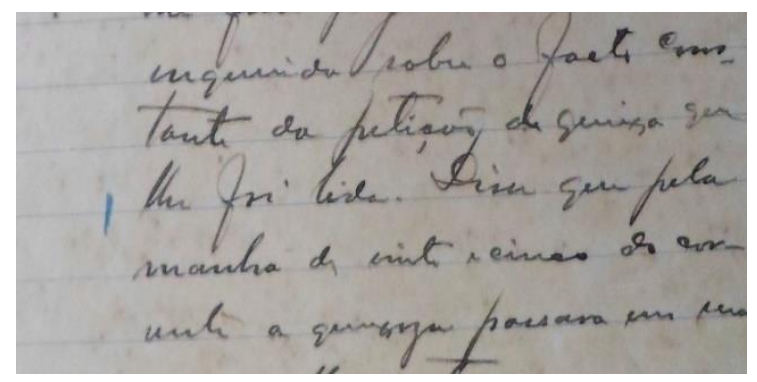

Fonte: Processo crime de roubo e estupro de Maria Francisca de Jesus e Maria dos Santos, 1913 / CEDOC / UEFS.

Fotografia: Damares Oliveira de Souza.

Figura 14 - Fól. 18v: Destaque para marcações com lápis de cera de cor azul
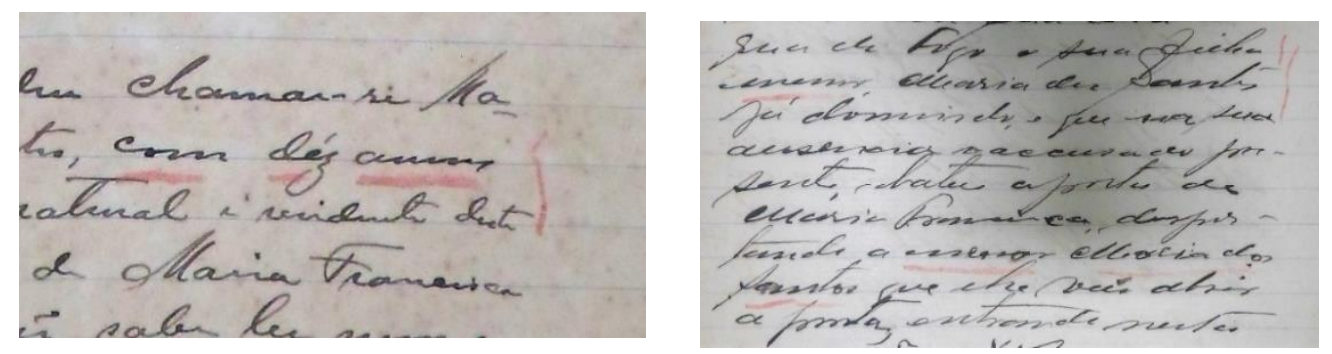

Fonte: Processo crime de roubo e estupro de Maria Francisca de Jesus e Maria dos Santos, 1913 / CEDOC / UEFS. Fotografia: Damares Oliveira de Souza.

Figuras 15 e 16 - Fóls. 12r e 28r: Destaque para marcações com lápis de cera de cor vermelha 


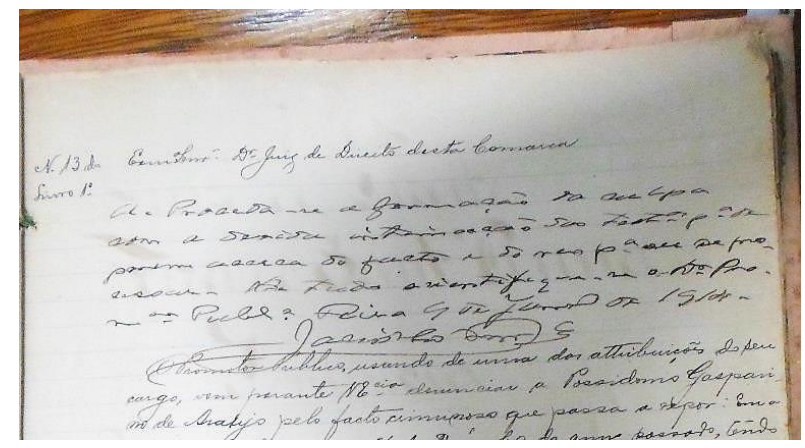

Fonte: Processo crime de roubo e estupro de Maria Francisca de Jesus e Maria dos Santos, 1913 / CEDOC / UEFS.

Fotografia: Damares Oliveira de Souza.

Figura 17 - Fólio 3r: Destaque para fólio sem numeração

O texto do processo crime de Maria Francisca e Maria dos Santos foi escrito em letra cursiva. Observamos a caligrafia de quatro escrivães, os quais foram identificados, também, através de suas assinaturas, como pode ser constatado a seguir:

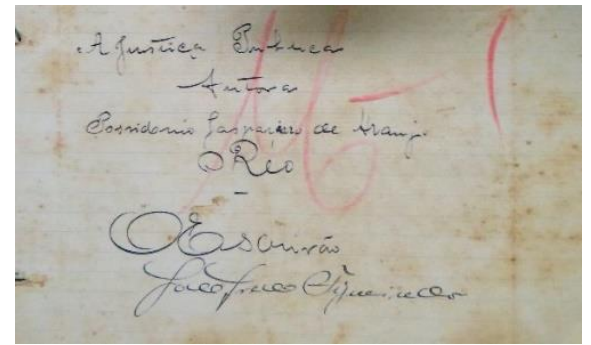

Figura 18 - Fól. $1 \mathrm{r}$

Escrivão: Godofredo Rebello de Figueiredo

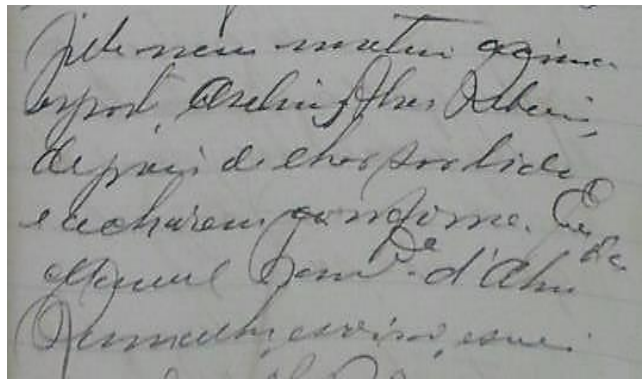

Figura 20 - Fól. 29r

Escrivão: Manuel Francisco de A. Ramalho

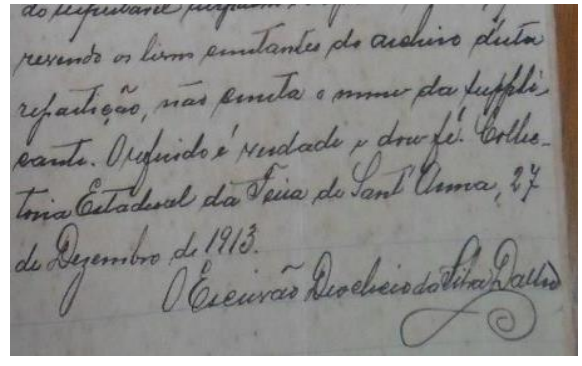

Figura 19 - Fól. $7 \mathrm{r}$

Escrivão: Deoclecio da Silva Daltro

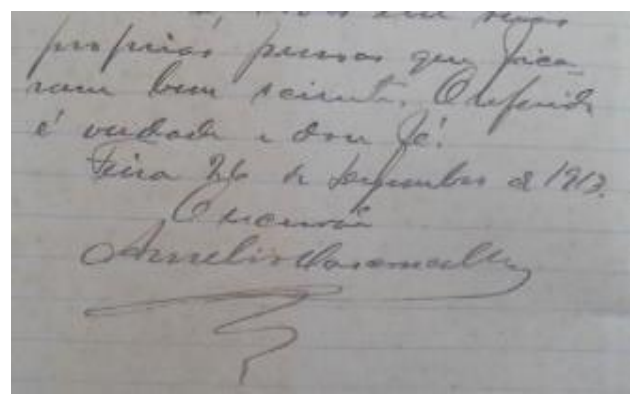

Figura 21 - Fól. 5v

Escrivão: Aurelio Vasconcellos

Outro aspecto considerável é a presença de abreviaturas. Desde a época romana, abreviar palavras é um mecanismo extremamente conhecido e utilizado para acelerar a escrita e economizar material empregado, como papel e tinta. De acordo com Spina (1977), na Idade Média o sistema de abreviaturas passou a ser muito usado, 
pois os materiais de escrita eram raros e, em razão disso, tinham preços elevados. Assim, com o uso das abreviaturas cada vez mais frequente, surgiram dificuldades para a leitura dos textos manuscritos, pois nem todos sabiam identificar a que palavras tais abreviaturas se referiam, razão pela qual Spina (1977, p. 44) afirma que o conhecimento das abreviaturas é "a chave para a interpretação paleográfica".

Para classificação das abreviaturas, tomamos como base os seis tipos definidos por Spina (1994, p. 45), bem como por Flexor (2008), os quais descrevemos e apresentamos, respectivamente, com os exemplos encontrados no processo crime, a saber:

i) Abreviatura por sigla: caracteriza-se pela representação da palavra por sua letra inicial maiúscula. Esse tipo ocorre de duas formas - quando indicada apenas por uma letra (sigla simples) ou ainda com a apresentação de duas letras iguais, indicando o plural desta palavra (sigla reduplicada). No entanto, identificamos casos apenas da sigla simples.

Quadro 1 - Abreviaturas por sigla

\begin{tabular}{|c|c|c|c|c|}
\hline $\begin{array}{l}\text { FAC- } \\
\text { SÍMILE }\end{array}$ & $\begin{array}{l}\text { ABREVIA- } \\
\text { TURA }\end{array}$ & $\begin{array}{c}\text { DESDOBRAMEN- } \\
\text { TO }\end{array}$ & OCORRÊNCIAS & $\begin{array}{l}\text { QUANTI- } \\
\text { DADE }\end{array}$ \\
\hline & N. & Número & f. 3 r, 1.1 ; f. 52 r, 1.6 & 2 \\
\hline & V & Vossa & $\begin{array}{l}\text { f. } 3 \text { r, 1. 12; f. } 3 \mathrm{v}, 1.4 \text {; f. } \\
\text { 5r, 1. 20; f. 6r, 1. 15; f. } 7 \text { r, } \\
\text { l. } 14 .\end{array}$ & 5 \\
\hline & A. & Arthur & $\begin{array}{l}\text { f. 4, l. 16; f. 9r; f. 12r; f. } \\
\text { 13r; f. 14r; f. 16r; f. 17r; } \\
\text { f. 18r; f. 19r; f. 20r (do f. } \\
\text { 9r ao f. 20r, esta } \\
\text { abreviatura aparece na } \\
\text { margem direita, no } \\
\text { sentido vertical). }\end{array}$ & 10 \\
\hline & P. & Pede & $\begin{array}{c}\text { f. 5r, 1. 26; f. 6r, 1. 20; f. } \\
\text { 7r, 1. } 19 .\end{array}$ & 3 \\
\hline & p. & próximo & f. $34 \mathrm{v}, 1.30$. & 1 \\
\hline & J. & Jonas & f. 38 r, $1.18 ;$ f. 43 r, 1.24 & 2 \\
\hline & P. & Para & $\begin{array}{l}\text { f. } 3 \mathrm{r}: 1.4 \text { e } 1.5 ; \text { f. } 3 \mathrm{v}, 1.4 \\
\text { f. } 33 \mathrm{v}, 1.23 ; \text { f. } 37 \mathrm{r}, 1.9 ; \text { f. } \\
42 \mathrm{r}, 1.9 ; 43 \mathrm{v}, 1.27 ; \text { f. } \\
44 \mathrm{v}, 1.2 .\end{array}$ & 8 \\
\hline
\end{tabular}




\begin{tabular}{|c|c|c|c|c|}
\hline 2 & q. & que & $\begin{array}{l}\text { f. } 44 \mathrm{v}: 1.15 \text { e } 1.17 \text {; } \\
\text { f. } 45 \mathrm{r}, 1.3 .\end{array}$ & 3 \\
\hline $\begin{array}{l}\text { Fonte: Process } \\
\text { Elaboração: D }\end{array}$ & oo e & & & Total $=34$ \\
\hline
\end{tabular}

ii) Abreviatura por suspensão ou apócope: consiste na supressão dos elementos finais do vocábulo, em que se utiliza o ponto (.) como signo abreviativo.

Quadro 2 - Abreviaturas por apócope

\begin{tabular}{|c|c|c|c|c|}
\hline FAC-SÍMILE & $\begin{array}{l}\text { ABREVIA- } \\
\text { TURA }\end{array}$ & $\begin{array}{l}\text { DESDOBRA- } \\
\text { MENTO }\end{array}$ & OCORRÊNCIAS & $\begin{array}{l}\text { QUANTI- } \\
\text { DADE }\end{array}$ \\
\hline & Cod. & Codigo & $\begin{array}{l}\text { f. } 44 \text { r, l. } 13 \text {; f. } 45 \text { r, } 1.8 \text {; f. } 3 r \text {, } \\
\text { l. } 32 \text {; f. } 44 \text { r, l. } 13 \text {, f. } 45 \text { r, 1. } 8 .\end{array}$ & 5 \\
\hline & test. & testemunhas & f. $5 r, 1.25$. & 1 \\
\hline & Es. & Escrivão & f. $34 \mathrm{v}, 1.10$. & 1 \\
\hline & cida. & cidade & f. $35 r, 1.9$. & 1 \\
\hline & art. & artigo & f. $52 \mathrm{r}, 1.6$. & 1 \\
\hline nte: Pro & bo e estupro & & & Total $=9$ \\
\hline
\end{tabular}

iii) Abreviatura por contração ou síncope: é o tipo de abreviatura em que ocorre supressão de letras no meio do vocábulo. Para facilitar a identificação, conservam-se letras intermediárias, chamadas de características.

Quadro 3 - Abreviaturas por síncope

\begin{tabular}{|c|c|c|c|c|}
\hline $\begin{array}{c}\text { FAC- } \\
\text { SÍMILE }\end{array}$ & $\begin{array}{c}\text { ABREVIA- } \\
\text { TURA }\end{array}$ & $\begin{array}{c}\text { DESDO- } \\
\text { BRAMENTO }\end{array}$ & OCORRENCIAS & $\begin{array}{c}\text { QUANTI- } \\
\text { DADE }\end{array}$ \\
\hline Sr & Senhor & $\begin{array}{c}\text { f. } 5 \mathrm{r}, 1.8 ; \mathrm{f} .6 \mathrm{r}, 1.2 ; \mathrm{f} .7 \mathrm{r}, 1.1 ; \mathrm{f} .20 \mathrm{r}: \\
\text { 1. 31 e 1. 33; f. 20v, 1. 1; f. 20v, 1. 25; } \\
\text { f. 38r, 1. 6; f. 45v, 1. 14; f. 46r, 1. 18. }\end{array}$ & 10 \\
\hline Snr. & Senhor & f. $5 \mathrm{r}, 1.1$. & 1 \\
\hline
\end{tabular}




\begin{tabular}{l|l|l|c|}
\hline fs. & folhas & f. $45 \mathrm{r}, 1.5$. & 1 \\
\hline & & & Total $=12$ \\
\hline
\end{tabular}
Fonte: Processo crime de roubo e estupro (1914).
Elaboração: Damares Oliveira de Souza.

iv) Abreviatura por letras sobrescritas: este tipo de abreviatura ocorre na sobreposição da última ou das últimas letras da palavra. Constatamos que este foi o tipo de mais uso no corpus.

Quadro 4 - Abreviaturas por letras sobrescritas

\begin{tabular}{|c|c|c|c|c|}
\hline $\begin{array}{l}\text { FAC- } \\
\text { SÍMILE }\end{array}$ & $\begin{array}{l}\text { ABREVIA- } \\
\text { TURA }\end{array}$ & $\begin{array}{l}\text { DESDOBRA- } \\
\text { MENTO }\end{array}$ & OCORRÊNCIAS & $\begin{array}{l}\text { QUANTI- } \\
\text { DADE }\end{array}$ \\
\hline & Ex.mo & $\begin{array}{l}\text { Excelentissi } \\
\text { mo }\end{array}$ & $\begin{array}{l}\text { f. } 3 \text { r, 1. } 1 \text {; f. } 20 \mathrm{v}, 1.25 ; \text { f. } 21 \mathrm{r}, 1 . \\
\text { 9; f. } 22 \text { r, 1. } 2 \text {; f. } 27 \mathrm{v}, 1.8 \text {; f. } 38 \mathrm{v} \text {, } \\
\text { l. } 5 \text {. }\end{array}$ & 6 \\
\hline & Test. $^{\text {as }}$ & Testemunbas & f. $3 r, 1.6$. & 1 \\
\hline & $\mathrm{p}^{\mathrm{a}}$ & para & $\begin{array}{l}\text { f. } 3 \text { r: } 1.6 \text { e } 1.7 \text {; f. } 3 \text { v, } 1.4 \text {; f. } 33 \mathrm{v} \text {, } \\
\text { l. } 23 \text {; f. } 43 \text { r, } 1.27 .\end{array}$ & 5 \\
\hline & Art. ${ }^{\mathrm{OS}}$ & Artigos & f. $44 \mathrm{r}, 1.12 ;$ f. $45 \mathrm{r}, 1.8$. & 2 \\
\hline & Art. ${ }^{\mathrm{O}}$ & Artigo & f. 49r, 1. 26. & 1 \\
\hline & $\mathrm{D}^{\mathrm{r}}$ & Doutor & 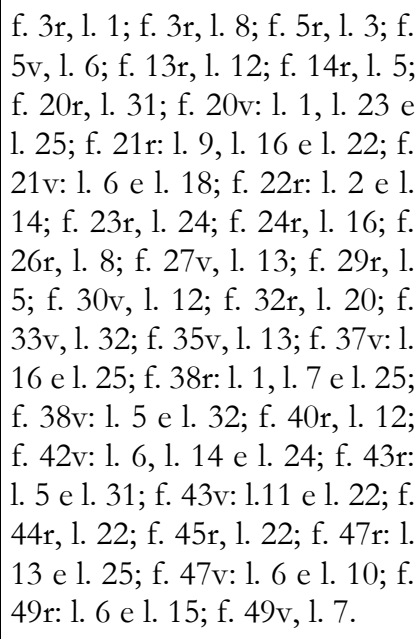 & 50 \\
\hline$n$ ? & Promo. ${ }^{\text {or }}$ & Promotor & $\begin{array}{c}\text { f. } 37 \mathrm{v}, 1.16 \text {; f. } 42 \mathrm{v}, 1.14 \text {; f. } 44 \mathrm{r}, \\
\text { 1. } 22 ; \text { f. } 47 \mathrm{r}, 1.25 \text {. }\end{array}$ & 4 \\
\hline
\end{tabular}




\begin{tabular}{|c|c|c|c|c|}
\hline uc & Publ. ${ }^{\circ}$ & Publico & f. $3 r, 1.9 ;$ f. $44 r, 1.22$. & 2 \\
\hline 18 & E.cia & Excelencia & f. 3 r, 1. 12; f. 3v, 1. 4. & 2 \\
\hline & Illmo & Illustrissimo & 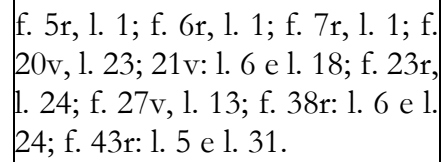 & 12 \\
\hline & $\mathrm{Sn}_{\mathrm{n}}^{\mathrm{S}}$ & Senhores & f. $5,1.3$. & 1 \\
\hline & Ferr $^{\mathrm{a}}$ & Ferreina & $\begin{array}{l}\text { f. 5r, l. 8; f. 23r, 1. 33; f. 25v, l. } \\
\text { 17; f. 26v, 1. 32; f. 29r, 1. 32; f. } \\
\text { 30v, 1. 32; f. 32v, 1. 9; f. 37r, 1. 24; } \\
\text { f. 37v, 1. 19; f. 38v, 1. 19; f. 40r } \\
\text { (esta abreviatura aparece na } \\
\text { margem direita, no sentido } \\
\text { vertical), f. 41r, l. 25; f. 42r, 1. 24; } \\
\text { f. 42v, 1. 17; f. 45r, 1. 16; f. 46r, 1. } \\
\text { 30; f. 47r, 1. 29; f. 51v, l. 2. }\end{array}$ & 18 \\
\hline & $\sup ^{e}$ & suplicante & f. $5 r, 1.14$ & 1 \\
\hline & $\operatorname{supp}^{\mathrm{e}}$ & supplicante & $\begin{array}{l}\text { f. } 5 \text { r: } 1.21 \text { e } 1.23 \text {; f. } 6 r: 1.16 \\
\text { e l. } 31 \text {; f. } 7 \text { r, l. } 15 \text {. }\end{array}$ & 5 \\
\hline & S. ${ }^{a}$ & Senhoria & $\begin{array}{l}\text { f. } 5 \text { r, 1. 20; f. } 6 r, 1.15 ; \text { f. } 7 r, 1 . \\
14 .\end{array}$ & 3 \\
\hline & $\mathrm{Se}^{\mathrm{r}}$ & Senbor & f. 5v, 1. 4; f. 19 r, 1.5. & 2 \\
\hline & Cel & Coronel & f. $6 r, 1.1$. & 1 \\
\hline & fl. ${ }^{2}$ & follhas & $\begin{array}{l}\text { f. } 14 \text { r, 1. 33; f. } 17 \text { v, 1. 26; f. 43r, } \\
\text { 1. } 21 \text {; f. } 43 \mathrm{v}, 1.25 \text {; f. } 44 \mathrm{r}: 1.21 \text {, } \\
\text { 1. } 23,1.25 \text { e 1. } 27 \text {; f. } 44 \mathrm{v}: 1.2 \mathrm{e} \\
\text { 1. } 3 \text {; f. } 45 \mathrm{r}, 1.5 .\end{array}$ & 11 \\
\hline & Pharma ${ }^{c o}$ & Pharmaceutico & f. $14 \mathrm{r}, 1.6$ & 1 \\
\hline
\end{tabular}




\begin{tabular}{|c|c|c|c|c|}
\hline Ne & $\operatorname{Alf}^{s}$ & Alferes & $\begin{array}{l}\text { f. 8r, l. 28; f. 10r, 1. 11; f. 11r, l. } \\
\text { 27; f. 14r, l. 4; f. 15v, l. 2; f. } \\
\text { 16v, 1. 24; f. 17v, 1. 8; f. 18r, l. } \\
\text { 30; f. 19r, 1. 10; f. 20r, 1. 12; f. } \\
\text { 20v, 1. 5; }\end{array}$ & 11 \\
\hline & $\operatorname{jan}^{O}$ & janeiro & f. $20 \mathrm{v}, 1.4$. & 1 \\
\hline & $\operatorname{Sen}^{r}$ & Senhor & $\begin{array}{l}\text { f. 21r, 1. 22; f. 38r, 1. 25; f. 38v, } \\
\text { 1. 5; f. 40r, 1. } 12 \text {; f. } 42 \text { v, l. 6; f. } \\
\text { 42v, 1. 23; f. 43r: } 1.5 \text { e 1. } 31 \text {; f. } \\
\text { 43v, 1. 11; f. 46v: } 1.6 \text { e 1. } 20 .\end{array}$ & 11 \\
\hline & $\mathrm{Sn}^{\mathrm{r}}$ & Senbor & $\begin{array}{l}\text { f. 11r, 1. 19; f. 14r, 1. 17; f. 20v, } \\
\text { 1. 23; f. 21r, 1. 9; f. 21v: 1. } 6 \text { e l. } \\
\text { 18; f. 22r: 1. 2 e 14; f. 23r, 1. 24; } \\
\text { f. 24r, 1. 14; f. 27v: 1. } 9 \text { e 13; f. } \\
\text { 32r, l. 20; f. 37v, l. 25; f. 38v, l. } \\
\text { 31. }\end{array}$ & 15 \\
\hline & Clos & Conclusos & 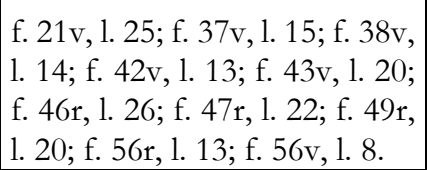 & 10 \\
\hline & Almda & Almeida & 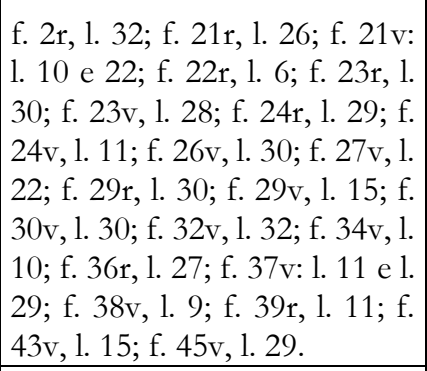 & 23 \\
\hline $\begin{array}{l}4 \\
0\end{array}$ & Franco & Francisco & 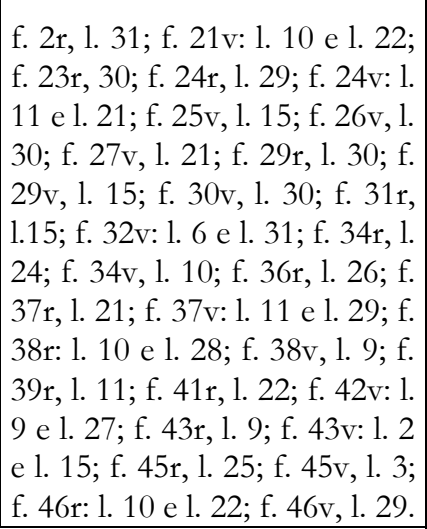 & 37 \\
\hline te: 1 & 00 & & & Total $=236$ \\
\hline
\end{tabular}


v) Abreviatura por letras numerais: constituem-se por abreviaturas com numerações, designativas de ordem, divisão e meses do ano.

Quadro 5 - Abreviaturas por letras numerais

\begin{tabular}{|c|c|c|c|c|}
\hline $\begin{array}{l}\text { FAC- } \\
\text { SÍMILE }\end{array}$ & $\begin{array}{l}\text { ABREVIATU- } \\
\text { RA }\end{array}$ & $\begin{array}{c}\text { DESDOBRAMEN- } \\
\text { TO }\end{array}$ & $\begin{array}{l}\text { OCORRÊN- } \\
\text { CIAS }\end{array}$ & $\begin{array}{l}\text { QUANTI- } \\
\text { DADE }\end{array}$ \\
\hline & $1^{\mathrm{a}}$ & Primeira & $\begin{array}{l}\text { f. } 3 \mathrm{v}, 1.11 ; \text { f. } 16 \mathrm{r}, 1 . \\
17 \text {; f. } 27 \mathrm{v}, 1.25 ; \text { f. } \\
48 \mathrm{v}, 1.23 \text {; f. } 52 \mathrm{r}, 1 \\
12 \text {, f. } 53 \mathrm{r}, 1.11 .\end{array}$ & 6 \\
\hline & $2^{a}$ & Segunda & $\begin{array}{l}\text { f. } 3 \mathrm{v}, 1.1 \text {; f. } 3 \mathrm{v}, 1.12 \text {; } \\
\text { f. } 3 \mathrm{v}, 1.12 ; \text { f. } 16 \mathrm{v}, 1 . \\
30 ; \text { f. } 29 \mathrm{v}, 1.18 ; \text { f. } \\
48 \mathrm{r}, 1.14 ; \text { f. } 48 \mathrm{v}, 1 . \\
24 .\end{array}$ & 7 \\
\hline & $3^{a}$ & Terceira & $\begin{array}{l}\text { f. } 3 \mathrm{v}, 1.13 ; \text { f. } 17 \mathrm{v}, 1 . \\
\text { 15; f. } 31 \mathrm{r}, 1.18 ; \text { f. } \\
48 \mathrm{r}, 1.18 ; \text { f. } 48 \mathrm{v}, 1 . \\
25 .\end{array}$ & 5 \\
\hline & $4^{\mathrm{a}}$ & Quarta & $\begin{array}{l}\text { f. } 3 \mathrm{v}, 1.14 ; \text { f. } 48 \mathrm{r}, 1 . \\
21 ; \text { f. } 48 \mathrm{v}, 1.26 .\end{array}$ & 3 \\
\hline & $5^{\mathrm{a}}$ & Quinta & $\begin{array}{l}\text { f. } 3 \mathrm{v}, 1.15 ; \text { f. } 48 \mathrm{r}, 1 . \\
25 ; \text { f. } 48 \mathrm{v}, 1.27 \text {. }\end{array}$ & 3 \\
\hline & $6^{a}$ & Sexta & f. $48 \mathrm{r}, 1.31$. & 1 \\
\hline & $7^{a}$ & Sétima & f. $48 \mathrm{v}, 1.1$. & 1 \\
\hline & $23^{\mathrm{a}}$ & Vigésima terceira & f. $44 \mathrm{r}, 1.21$. & 1 \\
\hline $23:$ & $33^{\mathrm{a}}$ & Trigésima terceira & f. $44 \mathrm{r}, 1.23$. & 1 \\
\hline $38:$ & $38^{\mathrm{a}}$ & Trigésima oitava & f. 44r, 1. 27. & 1 \\
\hline cess & de roubo e estl & & & Total $=23$ \\
\hline
\end{tabular}

vi) Abreviatura por signo especial de abreviação: tipo em que ocorre a presença de um sinal colocado no início, meio ou fim da palavra abreviada, indicando os elementos ausentes. 
Quadro 6 - Abreviatura por signo especial de abreviação

\begin{tabular}{|c|c|c|c|c|}
\hline $\begin{array}{l}\text { FAC- } \\
\text { SÍMI- } \\
\text { LE }\end{array}$ & $\begin{array}{c}\text { ABREVIATU- } \\
\text { RA }\end{array}$ & $\begin{array}{c}\text { DESDOBRAMEN- } \\
\text { TO }\end{array}$ & $\begin{array}{l}\text { OCORRÊN- } \\
\text { CIAS }\end{array}$ & $\begin{array}{c}\text { QUANTIDA- } \\
\text { DE }\end{array}$ \\
\hline$\rho$ & $\int$ & Parágrafo & $\begin{array}{l}\text { f. } 3 \text { r, } 1.32 \text {; f. } 3 \text { v: } 1.1 \\
\text { e } 1.2 \text {; f. } 48 v \text { : } 1.8 \text { e } 1 . \\
\text { 9. }\end{array}$ & 5 \\
\hline $\begin{array}{l}\text { Proc } \\
\text { ração: }\end{array}$ & $\begin{array}{l}\text { me de roubo e e } \\
\text { es Oliveira de } \mathrm{S}\end{array}$ & 914). & & Total $=5$ \\
\hline
\end{tabular}

\section{CONSIDERAÇÕES FINAIS}

Sem dúvidas, realizar o estudo dos aspectos paleográficos do processo crime de roubo e estupro de Maria Francisca e Maria dos Santos, aliado às edições fac-similar e semidiplomática do mesmo, foi extremamente importante para o entendimento do texto e a sua completa interpretação filológica, uma vez que, segundo Berwanger e Leal (2008), a Paleografia é auxiliada pela Filologia, ao mesmo tempo em que lhe presta serviços.

Assim, ao realizarmos o estudo dos aspectos paleográficos no corpus em questão, pudemos identificar, além das características extrínsecas, expostas no quadro 1, a caligrafia de quatro escrivães, o que ficou comprovado, também, através de suas assinaturas. Quanto aos aspectos abreviativos, definidos e classificados em seis tipos (Spina, 1977), catalogamos o total de 319 abreviaturas, das quais, a maioria, quase 74\%, corresponderam a abreviaturas por letras sobrescritas.

Por fim, salientamos que o estudo paleográfico aqui apresentado, não se faz exaustivo, visto que, como já dito, o corpus deste trabalho é constante de uma dissertação de mestrado, defendida em março de 2018. Além das abreviaturas analisadas, faz-se de interesse da Filologia tudo o que se relaciona ao texto: as peculiaridades da escrita dos escrivães, como o traçado das letras e o grau de escolaridade; o vocabulário; as marcas d'água; o tipo de papel, dentre outros aspectos relevantes para a análise filológica, como as condições de produção documental.

\section{REFERÊNCIAS}

Acioli VLC. A escrita no Brasil Colônia: um guia para leitura de documentos manuscritos. $2^{\mathrm{a}}$ ed. Recife: UFPE / Fundação Joaquim Nabuco / Editora Massangana; 2003.

Andrade EA. Aspectos paleográficos em manuscritos dos séculos XVIII e XIX. Filologia e Linguística Portuguesa. 2009;10-11:149-172.

Berwanger AR, Leal JEF. Noções de paleografia e diplomática. $3^{\text {a }}$ ed. rev. e ampl. Santa Maria: Ed. UFSM; 2008.

Cambraia CN. Introdução à crítica textual. São Paulo: Martins Fontes; 2005.

Flexor MHO. Abreviaturas: manuscritos do século XVI ao XIX. $3^{\text {a }}$ ed. rev. aum. Rio de Janeiro: Arquivo Nacional; 2008. 
Queiroz RCR. Introdução metodológica. In: organizadora. Documentos do acervo de Monsenhor Galvão: edição semidiplomática. Feira de Santana: Universidade Estadual de Feira de Santana; 2007. p. 23-34.

Spaggiari B, Perugi M. Fundamentos da crítica textual. Rio de Janeiro: Lucerna; 2004.

Spina S. Introdução à edótica. São Paulo: Cultrix/Editora da Universidade de São Paulo; 1977. 